\title{
Overuse Injuries Correlated to the Mountain Bike's Adjustment: A Prospective Field Study
}

\author{
Manuel Sabeti-Aschraf ${ }^{1, *}$, Markus Serek², Mathias Geisler ${ }^{3}$, Max Imilian Schmidt ${ }^{1}$, Tom Pachtner ${ }^{1}$, \\ Antonia Ochsner ${ }^{1}$, Philipp Funovics ${ }^{1}$ and Alexandra Graf ${ }^{4}$ \\ ${ }^{1}$ Vienna Medical School, Department of Orthopaedics and Orthopaedic Surgery, AKH Wien; Währinger Gürtel 18-20, \\ 1090 Vienna, Austria \\ ${ }^{2}$ Orthopaedic Hospital, Gersthof Vienna, Wielemannsgasse 28, 1180 Vienna, Austria \\ ${ }^{3}$ Hospital of Lilienfeld, Lower Austria, Im Tale 2, 3180 Lilienfeld, Austria \\ ${ }^{4}$ Vienna Medical School, Section for Medical Statistics, AKH Wien; Währinger Gürtel 18-20, 1090 Vienna, Austria
}

\begin{abstract}
Mountain biking is an Olympic discipline and popular world wide. In comparison to conventional road cycling little is reported of overuse injuries. Especially, the set up of the mountain bike seems to play an important role in the onset of overused body regions. Aim of this investigation is to identify overuse injuries in competitive mountain bikers and correlate them with technical settings of their bikes.

This prospective field study consists of two phases analyzing volunteer competitive mountain bikers who were interviewed with a preformed questionnaire. In Phase 1 overused body regions were identified. In Phase 2 riders were examined before and after the race for overused body regions which were correlated to the bike's adjustment. For this reason the athlete was sitting on a fixed mountain bike in riding position and the various distances were individually controlled.

169 competitors were analyzed of whom 87 had overuse injuries after the race. Most injuries concerned the lower back, the buttocks and the knee. There was a significant correlation between inadequate saddle-pedal distance and the incidence of knee pain $(p<0.038)$, and paraesthetic sensations in the hand $(p<0.023)$. The saddle inclination increased the incidence of pain in the buttocks $(p<0.014)$ ). Symptoms occurred more frequently in downhill $(p<0.0001)$ and uphill $(p<0.0007)$ passages.

Overuse injuries are frequently observed in competitive mountain bikers. Certain detailed adjustments have a direct impact on the incidence of overuse injuries in the competitive mountain bike cyclist.
\end{abstract}

\section{BACKGROUND}

Since the beginning of off-road cycling in the 1970s, off-road biking has become tremendously popular all over the world and attracts athletes of all ages, even children [1]. Although mountain biking has been an Olympic discipline since 1996, little is known about the incidence of injuries within this sport.

In the beginning, front suspension forks improved the shock absorbing characteristics tremendously (so-called "Hardtails"). In the past fifteen years, rear and front suspended bikes are available (so-called "Fullys"). In mountain biking marathons, the most popular form of competitive mountain biking, participants ride increasingly more Fullys. Full suspension bikes have become increasingly more popular with competitive athletes due to their increased shock absorbing ability. In addition, the suspension increases the interface between ground surface and tires in rough terrain and thus allows higher speed. Both effects together postpone

*Address correspondence to this author at the AKH- Wien Orthopädie, Währinger Güretl 18-20, 1090 Vienna, Austria; Tel: 0043140400 4060; E-mail: manuel.sabeti-aschraf@meduniwien.ac.at the onset of fatigue and lack of concentration. One of the main issues in mountain biking overuse injuries seems to be the related to vibration and shock. In uphill passages the rider has time to react on rough terrain, although vibrations cannot be avoided completely, they are reduced when compared to downhill sections of the trails. In these situations, riders are probably not prepared for strong shocks and the probability to be involved in accidents rises. Due to vibrations and shocks it seems obvious that especially in the lumbar spine, in the hands, the knees and the feet, overuse injuries may occur in high numbers. In road cycle racing, overuse injuries are reported of the median nerve in the carpal tunnel [2], this is observed in off-road motorcyclists as well [3] the ulnar nerve in Guillon's Canal [2] the perineal nerve, the cervical [4] and lumbar spine and in the knees. Although recent studies have reported injuries in recreational and competitive off-road biking [5-10] little is known about overuse injuries in competitions [4, 8-10]. In 2002 it was recommended [11] that further investigations in mountain biking should address overuse injuries. To be seated ergonomically, individual adjustment of the mountain bike (MTB) to the rider is mandatory and should centre the rider's barycentre on the bike. Otherwise, the rider faces early onset 
of overuse syndromes especially in steep up- and downhill passages. In uphill passages the centre of gravity is moved backwards subsequently overloading the buttocks and the lumbar spine. The rider necessarily flexes the low back and pulls his weight to the front with the handlebars to maintain a balanced position and avoid a lift-off of the front wheel. Both, the pulling of the arms and the hyper-flexed position in the lumbar spine may result in low back problems. To maintain a more comfortable position, especially to improve the tolerance to shock and vibration, riders often ride the bike in a standing position. The disadvantage of pedalling in this position is the high consumption of energy and the reduced traction of the rear tire in uphill passages. Hence a competitive rider will avoid the standing position as long as possible to conserve energy.

In contrast, during downhill passages the rider has to absorb most of his load with the upper extremities. To restore a stable position on the bike, the wrist is set in a slightly dorsiflexed position and the elbows are slightly extended to move the barycentre as far back as possible.

Independent of the track conditions, the bike itself may either support the rider's comfort or force him into one of the above described riding positions. Some adjustments, which are important to keep the rider in a balanced and comfortable, and therefore, energy saving position, will later be described. Only few reports dealing with the proper adjustment of bikes are available. Especially, regarding the intentions of the athlete in terms of a rather competitive or rather comfortable setting [12] of the used MTB. The saddle-pedal-distance is the crucial part in the bicycle adjustment. All other adjustments should be done subsequently.

A correctly adjusted saddle-pedal-distance helps the athlete to remain in a sitting position as long as possible. Hence, most of his weight is carried by the buttocks. Moreover, the recruitment of available muscles for pedalling is optimized and reduces the femoro-patellar pressure in the nearly extended knee compared to a low saddle position. If the saddle is fixed in a position that is too high, the upper ankle joint has to be hyper-flexed, which exhausts the flexor muscle group. Additionally, the pressure on the buttocks increases the loads of this body region. After having the saddle set to the correct position, the angulation of the saddle needs to be adjusted. A horizontally fixed saddle is found on most bikes, which helps to distribute the load equally onto the buttocks. A slightly anteriorly inclined saddle brings more loads to the siatic bone and takes load away from the pubic area. If the inclination is too steep the rider tends to slide downwards and has to push himself back into position with knees and arms. On the one hand, this position is energy consuming and on the other hand it brings the low back into a too upright position which leads to a decreased ability to absorb shocks. In contrast, a posteriorly inclined saddle position overloads the pubic area. This is uncomfortable for both, females and males, and brings the rider into an inefficient pedalling position.

Adjustment of the distance of the handlebar to the saddle (saddle-handlebar-distance) is the third step. Depending on the demands of the rider, the height of the handlebar in comparison to the saddle can be lowered to obtain a competitive setting. In this position the rider remains in an aerodynamic position and the front tire tends to keep better contact with the trail surface in steep uphill passages. Additionally, due to the flexion of the lumbar spine, the gluteus muscles can be recruited for even more efficient pedalling. On the other hand, with handlebars that are significantly raised above the saddle, the rider has less flexion in the low back, less pressure on the upper extremity and is thus in a more comfortable position. Aim of this study is to identify overused body regions in competitive mountain bikers. We attempt to correlate the onset of pain with situations on the track like up- or downhill passages. Additionally, we analyzed whether the incidence of overuse injuries has a distinct correleation to the mountain bike's adjustment to the rider. So far, according to our knowledge, no other study has analyzed the incidence and the localization of overused body regions for competitive mountain bikers and correlated these findings with the bike's adjustments.

\section{METHODS}

This study consists of two separately investigated mountain bike races analysing athletes who volunteered to participate in our study. All athletes gave their oral consent before being included in this trial. The first investigation was planned to detect the overused body regions and was carried out at the "Rolling Stones"- Mountainbike Challenge at Stattegg, Austria. The race belongs to the Austrian Mountain Bike Cup and to the World Series Races. In addition to detecting overuse injuries in competitive mountain bikers, we analyzed the correleation between injury and various study parameters (e.g. race distance, training set-up or pain during the race).

The second study was performed at the "Babenberger Trophy" in order to get more detailed information about the influence of specific bike adjustments on overused body regions. The Babenberger Trophy was the official Austrian Mountain Bike Championship.

Both races were supported by the Union Cycliste Internationale. In both races various categories according to the distances called small, medium and large were available. The riders participated in different categories according to their age or their riding ability. The riders were informed about this investigation in the event schedule, with flyers at the registration office and by the local speakers. For the second race, the study was additionally announced online. The study investigators were positioned in the start and finish areas. Participating athletes were interviewed with preformed questionnaires including demographic data, chosen distance and type of mountain bike (Hardtail, Fully). Various anatomical regions including cervical-, thoracic and lumbar spine, hand and wrist, elbow, shoulder, buttocks, hip, knee, and foot were evaluated separately using the Visual Analogue Scale (VAS) for pain assessment. The occurrence of pain during training was also noted.

\section{Rolling Stones}

In this race, the limited range of motion of painful regions was noted additionally to the data mentioned above. Furthermore we asked if the training set-up itself was changed due to pain, if pain increased during competition and if any kind of therapy had already been administered. It was also recorded if a rider had changed his bike due to pain. 
Finally, the stage in which pain occurred during the race including uphill, downhill or in a flat area was registered.

\section{Statistical Analysis}

For the two body regions with the largest incidence of overuse injuries (lumbar spine, knee) the influence of the measured parameters on the occurrence of overuse injuries after the race was investigated. Therefore stepwise logistic regression models were calculated for the target variable "pain in lumbar spine after the race" (binary outcome: yes or no) as well as for the target variable "pain in knee after the race" (binary outcome: yes or no). Both logistic regression models were accounting for the measured parameters: age, weight, height, quantity of technical or endurance training (in hours per month) as well as suspension of bike (Hardtail vs. Fully) and pain during downhill, uphill and flat passages of the race (yes vs. no) as covariables. For all other body regions (e.g. hand or shoulder) due to the small occurrence of overuse injuries after the race no such analysis was performed.

\section{Babenberger Trophy}

The second study was planned in order to get more detailed information about the influence of bike adjustments on the incidence of overused body regions. Therefore voluntary athletes were examined before and immediately after the race according to the anatomical regions analyzed in Phase 1 with a preformed questionnaire (Fig. 1). Each rider was examined at the author's desk presenting himself and the bike. The examiners then analyzed the rider and the bike separately, at first, and then the rider sitting on the bike. To evaluate the bicycle adjustment and the type of setting (race, sporty or comfort), the inclination of the saddle (anteriorly-, posteriorly- horizontally- orientated), the saddle-pedaldistance and the saddle-handlebar-distance were noted. For pain assessment the VAS was used. Additionally, paraesthetic sensations in the hand and fingers were recorded by examining each finger of both hands taking the innervation area of the median-, the radial-, and the ulnar nerve into consideration.

\section{Statistics}

Based on the deviation of the distribution of VAS scores from the normal distribution we performed a stepwise logistic regression model for the binary outcome "pain" ("no pain": VAS $=0$ vs. "pain": VAS $>0$ ) in each of the investigated anatomical regions (pain in lumbar spine, knee and buttocks). These logistic regression models accounted for pain before the race, age, weight, height and the different requested bike adjustments (see questionnaire). For the paraesthetic sensations the same analysis was performed.

In both studies, all p-values smaller than 0.05 were considered as statistically significant. All analyses were performed using the SAS 9.1. System.

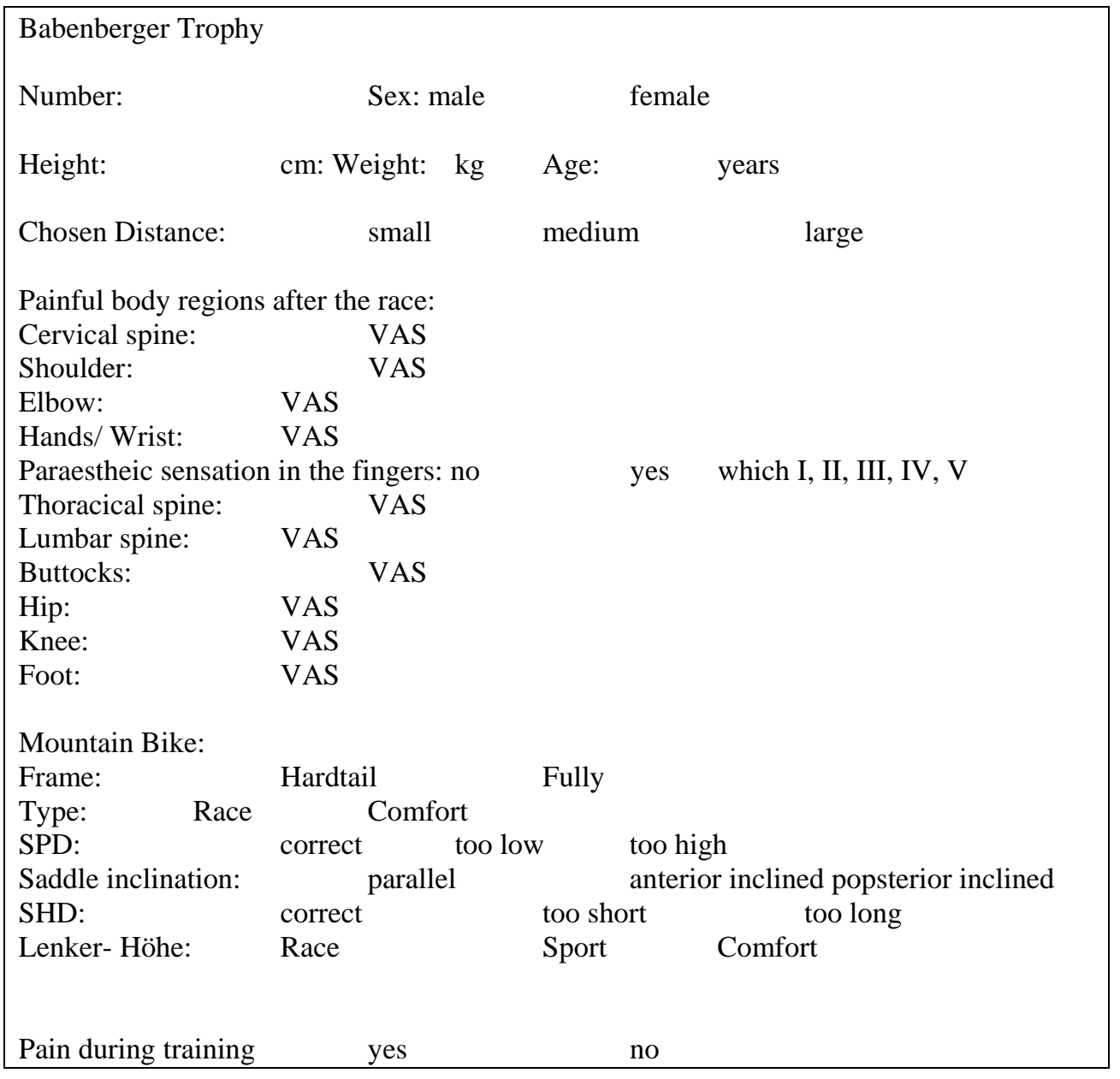

Fig. (1). Questionnaire of the Babenberger Trophy. 


\section{RESULTS}

Alltogether, 167 athletes (15 female, 153 male) were analyzed in Phase 1 and 2. 87 of them reported having at least one painful body region after the race. The average age of the riders was 32.4 years with a mean weight of $72.1 \mathrm{~kg}$ and a mean height of $176.9 \mathrm{~cm}$. Detailed demographic data of the analyzed riders according to the race are given in Table 1. Overall, 70 riders participated in the short distance, 73 in the medium and 26 in the long distance. Detailed data of the chosen distance in the race is listed in Table 2. In total, the predominately overused body region was, in 63 records the lower back, followed by the buttocks with 16 and the knee with 15 records.

\section{The Rolling Stones}

After the race, 46 (31 rode Hardtails, 15 Fullies) of the evaluated 94 cyclists complained about at least one painful body region. The predominant painful region was the lumbar spine (32 racers) followed by the knee (10 racers). In 5 cases the hand, 4 times the cervical spine and twice the thoracic spine, the foot and the shoulder were reported as being painful. 24 racers consulted a doctor for therapy. 13 athletes previously changed their training set-up to prevent overuse syndromes by increasing technical training and adaptation of the saddle-handlebar-distance. 6 racers had changed their bikes due to pain.

\section{Overuse Injuries in the Lumbar Spine}

32 racers complained about pain in the lumbar spine. All racers had unrestricted range of motion after the race except for one. 8 of the 32 riders reported pain in steep uphill passages, 25 reported pain in steep downhill passages and 4 complained about pain throughout the race. A statistically significant influence of pain in uphill passages $(p=0.0007)$ and pain in downhill passages $(p<0.0001)$ on pain in lumbar spine after the race was found. Clearly, as expected, the risk of having an overuse injury in the lumbar spine is larger for racers having pain already during the race. However, no significant influence of racing distance, type of bike, training set-up, age, height or weight was found.

15 athletes were already in therapy for pain relief before the race including Yoga, local antirheumatics, massage, muscle building, and other.

\section{Overuse Injuries in the Knee}

10 athletes had pain in the knee joint immediately after the race. Again a significant influence of pain in downhill passages $(p=0.0082)$ on pain in knee after the race was found. 9 of the 10 athletes had pain in downhill passages. Interestingly, a significant influence of height was found. Taller riders had significantly less pain in the knee $(\mathrm{p}=0.0153)$. However, again no significant influence of racing distance, type of bike, training set-up, age or weight was found.

\section{Other Body Regions}

The other body regions were not reported to be painful during or immediately after the race.

\section{Correlation of Pain and Track Condition}

Of the 94 riders, 38 reported the on-set of pain in downhill passages. 13 riders felt pain in uphill passages and in 6 cases pain was evident on flat terrain. Independently of the site of pain, the onset of symptoms in downhill $(p<0.0001)$ and uphill $(\mathrm{p}<0.0007)$ passages and symptoms after the race were highly statistically significant. More precisely, also for the pain in the knee after the race, a statistical significance correlation to symptoms in downhill passages was observed $(\mathrm{p}<0.0082)$.

\section{The Babenberger Trophy}

This prospective study protocol was completed by 73 athletes of whom 45 reported about at least one overused body region after the race. As in the Rolling Stones, the predominantly painful body region was the lower back. Only a few overuse injuries were found in the knee. In a contrast to the Rolling Stones race, the buttocks were also frequently reported to be painful. 16 athletes reportedly had pain in the buttocks after the race. Detailed data of the various painful body regions according to the time points "before" and "after" the race is listed in Table $\mathbf{3}$.

Fully suspended bikes were used in 70 cases as compared to 3 Hardtail bikes. Three bikes had a comfort set-up with the handlebar higher than the saddle. In 12 cases the saddlehandlebar-distance was too long regarding anatomically correct adjustments. Concerning the saddle adjustment, 66 were correctly mounted, one was too high and six too low. The

Table 1. Demographic Data of Analysed Athletes

\begin{tabular}{|c|c|c|c|c|c|}
\hline Race & Athletes & $\operatorname{Sex} \mathbf{m} / \mathbf{f}$ & Age (Years) & Height (cm) & Weight (kg) \\
\hline Rolling Stones & 94 & $85 / 9$ & 32.39 & 177.76 & 70.46 \\
\hline
\end{tabular}

Table 2. Number of Starters According to the Chosen Distance

\begin{tabular}{|c|c|c|c|}
\hline Race & Short & Medium & Large \\
\hline \hline Rolling Stones & 39 & 31 & 24 \\
\hline Babenberger & 31 & 41 & 1 \\
\hline
\end{tabular}


Table 3. Overused Body Regions before and after the Race for the Babenberger Trophy

\begin{tabular}{|c|c|c|c|c|c|c|c|c|c|}
\hline & Cervial Spine & Thoracic Spine & Lumbar Spine & Hand & Elbow & Shoulder & Buttocks & Hip & Knee \\
\hline Before & 0 & 0 & 6 & 0 & 0 & 0 & 0 & 0 & 0 \\
\hline
\end{tabular}

saddle was fixed in a horizontal position in 58 cases, in 13 cases we recorded an anteriorly inclined saddle and in one case a posteriorly inclined saddle.

\section{Overuse Injury in Lumbar Spine}

6 racers complained about pain in the lumbar spine before the race as compared to 32 after the race (see Table 3). No significant influence of bike adjustments on pain in the lumbar spine after the race was found.

\section{Overuse Injury in Knee}

Only 5 racers complained about pain in the knee after the race (see Table 3). However, a significant result for saddlepedal-distance on the incidence of overuse syndromes in the knee was found $(p=0.038)$. Riders with the saddle-pedaldistance was adjusted correctly had a lower chance to get pain in the knee after the race.

\section{Overuse Injury in the Buttocks}

16 racers complained about pain in the buttocks after the race (see Table 3). A significant influence of the inclination of the saddle on the occurrence of pain in the buttocks was found. Riders with horizontally mounted saddles had significantly less pain $(p=0.014)$ than athletes with an either anteriorly or posteriorly inclined saddle.

\section{Paraesthetic Sensations}

Riders with a correctly adjusted saddle-pedal-distance had significantly less paraesthetic sensations in their hands than athletes riding on bikes with either too high or too low adjusted saddles $(\mathrm{p}=0.023)$. More precisely, there was a significant influence of an incorrect saddle-pedal-distance on paraesthetic sensation in the inervation area of the median nerve $(\mathrm{p}=0.025)$.

No other significant influences of the bike adjustment on the anatomical regions or paraesthetic sensations were found from the analyses.

\section{DISCUSSION}

In this investigation a significant correlation between inappropriate mountain bike adjustments and the occurrence of overuse syndromes in competitive mountain bikers was found. Paraesthetic sensations in the hand, pain in the lumbar spine, the knee and the buttocks were identified as the predominant overused body regions reported by athletes.

The inclination of the saddle and the saddle-pedal distance were responsible for two thirds of the recorded syndromes of Phase 2 of our investigation. Hence, the saddle position is obviously the crucial point in the bike's adjustment, affecting the entire rider's position on the bike. The significant increase of overuse syndromes in up- and down- hill passages can be plausibly explained by the aggravation of symptoms due to the shift of loads. It was [13] found, that an anterior inclination of the saddle between 10 to 15 degrees reduces the occurrence of low back pain significantly. A significant correlation between type of bicycle, age or gender was not found. In our population, riders with a horizontally adjusted saddle had significantly less pain than those with an inclined one.

In Phase 1 of our investigation, 20 of 43 Hardtail riders reported pain in the lower back compared to 12 riders on Fullies. At this point, it should be mentioned that the small number of subjects is a limiting factor of our study, which may influence the comparison of overuse injuries regarding the use of Fullys or Hardtail bikes. Hardtail riders seem to be limited in their performance due to the increased exposure to vibrations. Another investigation [14] compared the effects of Hardtails versus Fullys in elite cyclists in rough outdoor conditions. It was stated that riders on Fullys were significantly faster with even pedal power in both populations. It was concluded that Fullys tend to be the better choice for cross country races. Regarding the results of Olympic- or World Championship- races there are mostly Hardtail riders found in the top ten. The explanation therefore might be that in these outstanding competitions riders would rather choose the lighter bike in order to perform at top level and to take earlier onset of pain into consideration. This theory is supported by Faiss et al. [15], reporting of nearly similar performance of riders on Fullys compared to those on the lighter Hardtails. Nevertheless, the reduction of the frequency of vibrations and their amplitude were significantly lower in the Fully population. Comparable data $[16,17]$ was also found by other authors. Overuse injuries are common in long distance cyclist. Road cyclists are predominately affected at the cervical and lumbar spine, the hand, and at the buttocks. Compared to road cyclists, mountain bikers ride in a more upright position. The saddle-handlebar distance is smaller in mountain bikes, which increases the agility and capacity for shock absorption. Lesions of the ulnar and median nerve are described in long distance bikers [2]. In our collective, the incidence of carpal tunnel like syndromes was significantly increased after the race and significantly related to the saddle-pedal-distance. A significant occurrence of paraesthetic sensations in the area of the media nerve was reported by off road motorcyclists [3] The authors did not correlate their findings with the adjustment of the motorbike.

The occurrence of knee pain in taller mountain bikers can not be explained by the obtained data. We will take this into consideration for the next study's design. Surprisingly, in Phase 2 even some highly experienced athletes including National Race- and Cup Winners had inappropriately adjusted bikes specifically regarding the low position of the 
saddle. We assume that the riders keep the saddle in this lower position on purpose, in order to bring the buttock backwards to avoid a fall over the handle bars in steep downhill passages of the race. According to our impression, the saddle-handlebar-distance plays another important role in the bike's adjustment. As the lumbar spine remains in a kyphotic position when riding the bike in a sitting position, a longer saddle-handlebar-distance might affect the incidence of lumbar pain. This could not be proved in our investigation due to on insuffiecent number of riders. Based on the obtained data we calculated the sample of 116 riders in total in which significant results can be expected.

Limiting for this investigation is that two different races were analyzed (Phase 1 and Phase 2). Due to the limited cooperation of the organizers of the first race, a consecutive study at the same race track was not possible. Secondly, the data was gained in a voluntary population. A prospective investigation of randomized samples could definitely improve the impact of the data. A correlation between certain painful anatomical regions and up- or downhill passages was not performed. The third limiting factor was the rather small number of examined athletes. Increasing the study group size will be one of our major issues for the following investigations.

\section{CONCLUSION}

Mountain biking seems to have a high potential to cause overuse injuries in competitive athletes. The predominantly reported painful body region was the lumbar spine, the buttocks and the knee joints, which significantly correlates with an inappropriately adjusted mountain bike. Specifically, the exact saddle adjustment to the athlete had significant effect on the onset of pain during or immediately after the race. More detailed conclusions can not be drawn at this point due to the small study group.

\section{DECLARATION}

This investigation represents the honest work of the authors listed above. The study was not supported financially and there is no conflict of interest in what so ever means.
The current manuscript was presented at the International Federation of Sports Medicine Congress in Barcelona Nov.2008.

\section{REFERENCES}

[1] Sabeti M, Serek M, Geisler M, Leitner I, Nehrer S. Sturzrisiko und sturzfolgen bei kindern im mountainbikerennen. Sport Orthop Sport Traumatol 2005; 21: 12-5.

[2] Patterson JM, Jaggars MM, Boyer MI. Ulnar and median nerve palsy in long-distance cyclists. A prospective study. Am J Sports Med 2003; 31: 585-9.

[3] Sabeti-Aschraf M, Serek M, Pachtner T, et al. The Enduro motorcyclist's wrist and other overuse injuries in competitive Enduro motorcyclists: a prospective study. Scand J Med Sci Sports 2008; 14: 582-90.

[4] Frobose I, Lucker B, Wittmann K. Overuse symptoms in mountainbikers: a study with an empirical questionnaire. Dtsch Z Sportmed 2001; 52: 311-5.

[5] Baker SP, O'Neill B, Haddon W. The injury severity score: An update. J Trauma 1976; 882-5.

[6] Gaulrapp H, Weber A, Rosemayer B. Injuries in mountain biking. Knee Surg Spors Traumatol Arthrosc 2001; 9: 48-53.

[7] Jeys LM, Cribb G, Toms AD, Hay SM. Mountain biking injuries in rural England. Br J Sports Med 2001; 35: 197-9.

[8] Kronisch RL, Chow TK, Simon LM, Wong PF. Acute injuries in off-road bicycle racing. Am J Sports Med 1996; 24: 88-93.

[9] Kronisch R. How to fit a mountain bike. Phys Sportsmed 1998, 26: 3.

[10] Pfeiffer RP, Kronisch RL. Off-road cycling injuries. An overview. Sports Med 1995; 19: 311-25.

[11] Kronisch RL, Pfeiffer RP, Chow TK, Hummel CB. Gender differences in acute mountain bike racing injuries. Clin J Sports Med 2002; 12: 158-64.

[12] Schwarz W. Mountainbike-fahrtechniken Österreichisches Journal für Sportmedizin 2004; 32-41.

[13] Salai M, Brosh T, Blankstein A, Oran A, Chechik A. Effect of changing the saddle angle on the incidence of low back pain in recreational bicyclists. Br J Sports Med 1999; 33: 398-400.

[14] Nishii T, Umemura Y, Kitagawa K. Full suspension mountain bike improves cycling performance. J Sport Med Phys Fitness 2004; 44: 356-60.

[15] Faiss R, Praz M, Meichtry A, Gobelet C, Deriaz O. The effect of mountain bike suspensions on vibrations and off-road uphill performance. J Sports Med Phys Fitness 2007; 47: 151-8.

[16] Nielens H, Lejeune T. Bicycle shock absorption systems and energy expended by the cyclist. Sports Med 2004; 34: 71-80.

[17] Dingerkus ML, Martinek V, Kolzow I, Imhoff A. Mountainbike related injuries and overuse syndromes. Dtsch Z Sportmed 1998; 49: $242-4$. 